\title{
Clinical distinction of cerebral haemorrhage and cerebral infarction
}

\author{
M. J. G. HARRISON \\ D.M., F.R.C.P. \\ Department of Neurological Studies, The Middlesex Hospital, Mortimer Street, London W1N 8AA
}

\begin{abstract}
Summary
The clinical features of 62 cases of computerized axial tomography scan-proved cerebral haemorrhage and 81 cases of cerebral infarction in the carotid territory have been compared in a retrospective case note survey. The clinical features which can help in making the distinction between haemorrhage and infarction at the bedside are discussed and compared with those of classical teaching which referred only to post-mortem proved cases.
\end{abstract}

\section{Introduction}

The classical descriptions of the clinical features of cerebral infarction and cerebral haemorrhage depended on information derived from post-mortem series (Aring and Merritt, 1935). Clearly any criteria derived from such studies might not be applicable to the distinction between haemorrhage and infarction in surviving patients.

This study has therefore looked at the clinical features of patients whose stroke has been positively identified by computerized axial tomographic (CAT) scanning as due to haemorrhage or infarction in the carotid territory.

\section{Method}

The notes of all patients with a diagnosis of cerebral infarction or cerebral haemorrhage in the carotid territory that had been confirmed by CAT scans were reviewed. Only cases investigated within 2 months of the acute neurological illness were studied to ensure that detailed information of the clinical presentation was available.

Difficulties can arise in the distinction between the CAT scan appearances of infarction and haemorrhage if the patient's first scan is taken more than 10 or 12 days after the clinical onset. The high density lesion of a haemorrhage may after such an interval become a low density lesion that mimicks the effect of infarction. Scans showing low density lesions which were not supported by an early scan were therefore carefully reviewed. If the lesion included the cerebral cortex a diagnosis of infarction was accepted. If the lesion was restricted to deep parts of the hemisphere the case was excluded, as the possibility of a deep resolving haematoma rather than an infarct could not be excluded. Cases with a CAT scan report of haemorrhagic infarction were also eliminated. In this way it was hoped to have groups of patients in whom the risk of an error in diagnosis seemed most unlikely.

Basic data that were extracted included age, sex, casual BP reading on admission, and a history from the patient or referring practitioner of pre-existing arterial hypertension, transient cerebral ischaemic attacks or prior strokes.

In the presentation of the stroke illness, note was taken of any headache, the presence and time of occurrence of nausea or vomiting, alteration of consciousness, epilepsy, and any subsequent deterioration.

From the account of the examination in hospital, information was sought about the level of consciousness, the nature of any focal deficit, neck stiffness, and any signs of brain stem damage or of decerebrate posturing. The presence of bruits over neck vessels was noted, as was the result of lumbar puncture.

\section{Results}

There were 62 examples of cerebral haemorrhage and 81 of infarction. Of the latter, 14 were considered separately since their CAT scans showed small capsular infarcts rather than larger, more superficially placed ischaemic lesions.

\section{Age}

The age structure of the groups is shown in Table 1. There were more patients over the age of 60 years in the group shown to have hemisphere infarction when compared with the group with haemorrhage $\left(\chi^{2}=7.6, P<0.01\right)$. The male to female ratio also differed, being 2.7:1 for major infarction and $1 \cdot 1: 1$ for haemorrhage $\left(\chi^{2}=5.5, P<0.05\right)$.

\section{Blood pressure}

Hypertension as defined by a casual BP reading 
TABle 1. Age structure of study population

\begin{tabular}{lccccccc}
\hline & & \multicolumn{3}{c}{ Age in years } & & \\
& $21-30$ & $31-40$ & $41-50$ & $51-60$ & $61-70$ & $71-80$ & Total \\
\hline A. Cerebral haemorrhage & 3 & 7 & 18 & 19 & 13 & 2 & $62(33 \mathrm{M}$, \\
B. Cerebral infarction & 4 & 6 & 12 & 13 & 26 & 6 & $67(49 \mathrm{M}$, \\
C. Capsular infarcts & 2 & 1 & 3 & 5 & 3 & 0 & $14(9 \mathrm{M})$ \\
& & & & & & $5 \mathrm{~F})$ \\
\hline
\end{tabular}

TABLe 2. Prevalence of hypertension in study population

\begin{tabular}{lccc}
\hline & $\begin{array}{c}\text { History of } \\
\text { elevated BP }\end{array}$ & $\begin{array}{c}\text { BP on admission } \\
>160 / 90 \mathrm{mmHg}\end{array}$ & Either \\
\hline A. Cerebral haemorrhage & $23 / 62(38 \%)$ & $44 / 62(71 \%)$ & $46 / 62(74 \%)$ \\
B. Cerebral infarction & $7 / 67(10 \%)$ & $21 / 67(31 \%)$ & $27 / 67(40 \%)$ \\
C. Capsular infarcts & $2 / 14(14 \%)$ & $3 / 14(21 \%)$ & $5 / 14(36 \%)$ \\
$\chi_{P}^{2}$ A $v$. B & 13.0 & 20 & 15.2 \\
$P$ & $<0.01$ & $<0.01$ & $<0.01$ \\
\hline
\end{tabular}

on admission of $160 / 90 \mathrm{mmHg}$ or greater, proved to have been detected more frequently in the cases of haemorrhage. A history of pre-existing hypertension was only obtained in approximately $50 \%$ of those with elevated arterial pressure on admission (Table 2).

\section{History (Groups A and B)}

Transient ischaemic attacks. The history taken on admission revealed evidence of a preceding transient ischaemic attack in 14 of the cases of cerebral infarction but in none of the patients shown to have a cerebral haemorrhage $\left(\chi^{2}=18.6, P<0.01\right)$. A completed stroke had occurred previously in one of the patients whose new stroke was due to infarction. Two patients with a haemorrhage had a history of a subarachnoid haemorrhage.

Headache and vomiting. The onset of the stroke was associated with headache in $43 \%$ of patients in Group B in whom the lesion proved to be an infarct, and in $56 \%$ of those with a cerebral haemorrhage, an insignificant difference. Lateralized headache was however more common among those with cerebral infarction (9 cases), only 2 victims of cerebral haemorrhage having such a headache. Ocular and facial pain was encountered in a small number in either group.

A history of brief coma with recovery of consciousness before admission to hospital was recorded in 5 of the patients with a haemorrhage and in one of those with an infarct. Persisting unresponsiveness from the onset was only seen with haemorrhage and $18 \%$ were so affected. Drowsiness was present in a further $44 \%$ of patients with cerebral haemorrhage but was also documented in $28 \%$ of those with infarcts.
Vomiting was also more common in haemorrhage (45\% of $7 \%$ of the infarcts $\left(\chi^{2}=21.8, P<0.01\right)$. In the cases of cerebral haemorrhage the vomiting was usually an early feature and was present at the onset of the illness or developed within a few hours in $76 \%$ of instances. No case of cerebral infarction was associated with vomiting at the onset.

Altered consciousness. Single seizures occurred in $6 \%$ of haemorrhages and in $9 \%$ of the infarcts.

\section{Neurological signs (Groups A and B)}

The examination revealed neck stiffness in $48 \%$ of the cases of haemorrhage but in only $4 \%$ of infarcts $\left(\chi^{2}=32.0, P<0.001\right)$. Signs of tentorial herniation (the appearance of a third nerve palsy or of progressive brain stem damage) were elicited in $34 \%$ of the cases of haemorrhage and in $10 \%$ of the patients with an infarct $\left(\chi^{2}=10.4, P<0.01\right)$. There was no difference in the interval between the onset of symptoms and the development of signs of herniation in the cases of haemorrhage and infarction. Decerebrate posturing developed in 6 patients with a haemorrhage. In one instance this occurred during the progressive changes of tentorial herniation. In 5 others decerebrate posturing developed in association with coma that had been present from the onset of the illness. All these 5 came from the group of 19 in whom the scan had shown blood in the ventricular system. Two patients with cerebral infarcts developed decerebrate postures on the second day of their illness.

Signs of a hemisphere lesion such as dysphasia, hemiparesis or hemianaesthesia were detected in all but one case of infarction and in $61 \%$ of the cases of haemorrhage. In 18 patients with cerebral haemorrhage there were bilateral 'upper motor 
neurone' signs, whilst in 6 , no focal signs could be detected.

A bruit over a neck vessel was heard in $19 \%$ of the patients with an infarct, but in none of the subjects with haemorrhage $\left(\chi^{2}=12 \cdot 4, P<0.01\right)$.

Cerebrospinal fluid. The cerebrospinal fluid had been examined in 30 cases of haemorrhage. Eighteen showed blood-stained fluid, 9 others showed xanthochromia or microscopic evidence of bleeding; 3 were clear. Confirmatory evidence of haemorrhage was thus seen in $90 \%$. Eleven cases of cerebral infarction had had a lumbar puncture. One sample was contaminated by a traumatic tap. The fluid appeared clear in all the other cases and the red cell count was $<20 \times 10^{12} / 1$ in each instance.

\section{Capsular infarcts (Group C)}

The 14 patients with small deep infarcts in the internal capsule showed a few differences from the larger group of cases with hemisphere infarcts. Thus vomiting and deterioration were each seen in only one patient. No patient had drowsiness, epilepsy or neck stiffness. Interestingly, none had had a transient ischaemic attack but 2 had a cervical bruit. The clinical picture was that of a lacunar infarct in 8 (pure motor hemiplegia, clumsy hand, ipsilateral weakness and ataxia). In 4, however, dysphasia was noted, and in 2, combined motor and sensory changes were seen. Angiography revealed internal carotid occlusion in one, and middle cerebral or intracranial branch occlusions in 2. Hypertension was detected in only $21 \%$.

\section{Discussion}

Aring and Merritt in 1935 reported on the differential diagnosis between cerebral haemorrhage and thrombosis based on a pathologically controlled series of 245 cases. They emphasized the greater incidence of hypertension, headache, vomiting, neck stiffness, coma, epilepsy, signs of herniation, and blood-stained cerebrospinal fluid in the fatal case of haemorrhage. Dalsgaard Nielsen in another postmortem controlled study in 1956 showed that cerebral haemorrhage, diagnosed clinically, could be confirmed pathologically in only $65 \%$ of instances and that a clinical diagnosis of cerebral infarction was correct in only $58 \%$. His own case material suggested that hypertension, youth, coma, and bilateral signs were diagnostic of haemorrhage in these fatal cases.

More recent investigation of the accuracy of the clinical distinction between cerebral haemorrhage and infarction based on death certification comparisons with pathologists' reports shows a similar error rate. Thus in 1966 Heasman and Lipworth found 36 haemorrhages among 206 cases of cerebrovascular disease diagnosed as infarcts, and 65 infarcts among 364 cases diagnosed in life as haemorrhages.

Another modern post-mortem study (Schaafsma, 1968) considered a particularly sudden onset of the illness, decerebrate spasms, hypothermia, and the ocular signs of brain stem compromise of clear distinguishing value for haemorrhage, and only a history of previous strokes of definite value in positively identifying infarction. Aring and Merrit's criteria (headache, vomiting, neck stiffness and hypertension) were considered less reliable though still in favour of haemorrhage. Schaafsma found fits more frequent with infarction in conflict with the earlier report.

These accounts all dealt with fatal cases. Whilst some of the mortality reflects accompanying systemic diseases and thrombo-embolic complications, the material is undoubtedly heavily biased by the large numbers affected by large haematomas or massive infarction.

The present study using computerized axial transmission tomographic scans for the identification of high density lesions due to haemorrhage and low density areas due to infarction, enables the clinical picture of non-fatal hemisphere infarction and haemorrhage to be studied.

Features that seem to help in the diagnosis of infarction prove to be a history of transient ischaemic attacks, but not of prior completed strokes, and the presence of bruits over major neck vessels. In favour of haemorrhage were vomiting, especially at onset, neck stiffness and coma from the onset.

Headache, transient loss of consciousness at the onset, and the finding of hypertension occurred with either cerebral haemorrhage or infarction, although all those features were more common in patients who had a haemorrhage. Similarly, clinical deterioration due to brain stem compromise secondary to tentorial herniation was more common with haemorrhages which act as mass lesions. The same process may complicate infarction, however, owing to oedematous swelling of a hemisphere that is the site of a large infarct. The timing of deterioration did not distinguish between infarction and haemorrhage but decerebrate posturing was more common in haemorrhage.

Blood-stained cerebrospinal fluid is diagnostic of haemorrhage if the puncture is not traumatic. Deterioration may follow lumbar puncture, however, and a clear fluid does not exclude a haemorrhage. In the absence of CAT scanning it represents a method of distinguishing haemorrhage in approximately $80 \%$ (Lee et al., 1975).

The cases of small capsular infarcts are also of interest. In 8 of the 14 the clinical picture was as described by Fisher in hypertensive patients with lacunar lesions. In this present study hypertension was less frequently found and angiography revealed 
evidence of atheromatous thrombo-embolism in several cases. That the same clinical picture of a small deep infarct can be seen with small vessel disease in the hypertensive (Fisher, 1969) and with disease of the carotid and middle cerebral artery (Weisberg, 1979) is clearly important in the management of such patients. The clinical picture of a lacunar stroke should perhaps not deter investigation by angiography if bruits or non-invasive investigation suggests the presence of neck vessel disease.

\section{Acknowledgments}

I am grateful to Professor John Marshall for continued support and the provision of facilities at the National Hospital, Queen Square, where this study was carried out. Dr B. Kendall kindly reviewed many of the CAT scans.

\section{References}

ArING, G.C.D. \& MerritT, H.H. (1935) Differential diagnosis between cerebral haemorrhage and cerebral thrombosis. Archives of Internal Medicine, 56, 435.

DalsgaARD-Nielsen, T. (1956) Survey of 1000 cases of apoplexia cerebri. Acta psychiatrica neurologica scandinavica, 30, 169.

FISHER, C.M. (1969) The arterial lesions underlying lacunes. Acta neuropathologica, 12, 1.

HeAsman, M.A. \& LiPWORTH, L. (1966) Accuracy of certification of causes of death. General Registrar's Office Studies on Medical and Population Subjects, No. 20. H.M. Stationery Office, London.

Lee, M.C., Heaney, L.M., Jacobsen, R.L. \& Klassen, A.C. (1975) 'Cerebrospinal fluid in cerebral hemorrhage and infarction. Stroke, 6, 638.

SchaAfSMA, S. (1968) On the differential diagnosis between cerebral haemorrhage and infarction. Journal of the Neurological Sciences, 7, 83.

WeISBERG. L.A. (1979) Computed tomography and pure motor hemiparesis. Neurology. Minneapolis, 29, 490. 